\title{
AVALIAÇÃO DE CAPACIDADE TECNOLÓGICA DE FORNECEDORES PARA APOIO AO DESENVOLVIMENTO DE PRODUTOS: ESTUDO DE CASO EM UMA MONTADORA DE ÔNIBUS
}

\section{TECHNOLOGICAL CAPABILITY ASSESSMENT OF SUPPLIERS TO SUPPORT PRODUCT DEVELOPMENT: CASE STUDY ON A BUSES AND COACHES MANUFACTURER}

\author{
Lucas Comerlato* lucas.comerlato@marcopolo.com.br \\ Carlos Alberto Costa** cacosta@ucs.br \\ Marcos Alexandre Luciano** marcos.luciano@ucs.br \\ *Marcopolo SA, Caxias do Sul, Brasil \\ **Universidade de Caxias do Sul (UCS), Caxias do Sul, RS
}

\begin{abstract}
Resumo: A gestão da cadeia de suprimentos tornou-se uma questão crítica no cenário de colaboração global. Não é mais esperado dos fornecedores apenas o provimento de mercadorias ou componentes pré-definidos, mas também soluções colaborativas de engenharia dentro do ciclo de desenvolvimento do produto. Assim, critérios de seleção para a definição de parceiros no fornecimento de uma solução devem ir além de suas competências de produção e capacidades logísticas, incluindo também as competências técnicas e de engenharia. Este, em certo sentido, pode ser considerado um cenário comum das grandes empresas, mas ainda é um desafio quando se trata de pequenas e médias empresas (PMEs). Este trabalho discute como PMEs podem ser incluídas em uma cadeia de suprimentos, levando-se em consideração parâmetros de avaliação para sua competência técnica relacionada com a colaboração de desenvolvimento de produto. Um conjunto de oito parâmetros foi proposto para avaliar a competência da equipe de engenharia, estrutura física e tecnológica e de responsabilidade legal. Para cada parâmetro valores e pesos foram definidos também. A avaliação foi aplicada a três empresas que são fornecedores de um fabricante de ônibus do Rio Grande do Sul, Brasil. Os resultados mostram que, mesmo quando se trata de parâmetros objetivos, os fornecedores e clientes podem ter percepções diferentes sobre as mesmas questões.
\end{abstract}

Palavras-chave: Cadeia de suprimentos. Desenvolvimento de produtos. Capacidade tecnológica.

Abstract: Supply chain management has become a critical issue in the global collaboration scenario. Suppliers are not expected to provide just commodities or pre-defined components, but also collaborative engineering design solutions within the product development cycle. Thus, the selection criteria for defining supply partner go beyond the production and logistic capabilities, but must also include technical and engineering competence. This, in some sense, can be considered a common scenario of big companies, but is still challenging when dealing with SME's. This work discusses how SME's can be included in a supply chains taking in consideration a set of parameters to assess their technical competence related to product development collaboration. A set of eight parameters has been proposed to assess the engineering team competence, physical and technological structure and further legal responsibility. For each parameter values and weights were defined also. The assessment was applied to three companies that are suppliers of a buses and coaches manufacturer from Rio Grande do Sul, Brazil. The results show that even when dealing with objective parameters, the suppliers and costumer can have different perceptions about same issues.

Keyword: Supply chain. Technical capability. Product development. 


\section{INTRODUÇÃO}

A Gestão da Cadeia de Suprimentos (Supply Chain Management) tem como fator chave a cooperação entre empresas para a organização de diferentes funções, como por exemplo: logística, marketing, desenvolvimento de produto, sustentabilidade, etc (BALLOU, 2004; HONG, 2006). Isso é complementado pelo relacionamento entre estas empresas, onde são estabelecidos os mecanismos de coordenação e práticas gerenciais para efetivar a cooperação e colaboração entre as diferentes unidades de negócios (ASSUMPÇÃO, 2003; SPEKMAN, KAMAUFF JR e MYHR, 1998).

As empresas montadoras na área automobilística possuem o conhecimento e competência sobre o desenvolvimento do seu produto final, i.e. veículo. Contudo, apesar de fazer parte do produto final, muitos dos componentes que integram o veículo, não são itens dos quais a empresa foca o seu know how para o desenvolvimento, como por exemplo, faróis, sinaleiras e lanternas; sistemas de transmissão e ar condicionado. Desta forma, é deixado a cargo dos fornecedores e/ou parceiros o aprimoramento dos sistemas, subsistemas e componentes (CHIESA, MANZINI e TECILLA, 2000; COUSINS e SPEKMAN, 2003; PEREIRA e GEIGER, 2005).

O envolvimento de fornecedores e/ou parceiros no processo de desenvolvimento de produtos normalmente ocorre quando se trata de um projeto estratégico para a empresa, e o parceiro escolhido possui competência essencial na área, ou seja, a capacidade de projetar e produzir com qualidade o componente ou sistema desejado. Segundo Amaral e Toledo (2000), a colaboração clientefornecedor no desenvolvimento de produtos é formada pela união de pessoas, recursos e mecanismos de interação das estruturas organizacionais de uma empresa cliente e uma fornecedora, engajadas num mesmo processo de desenvolvimento.

Alguns dos critérios comumente utilizados para a definição de fornecedores estão associados ao preço, qualidade, desempenho nas entregas, flexibilidade, análise financeira, análise de competências essenciais e aspectos relativos à 
segurança e meio-ambiente (FARIA e VANALLE, 2006). A aplicação desses fatores qualificadores na seleção de um fornecedor deve também considerar o porte da empresa e sua capacidade colaborativa, bem como a complexidade tecnológica do produto e/ou processo que está sendo adquirido.

Empresas de grande porte, como por exemplo, fornecedores globais, possuem normalmente condições de atenderem seus clientes no prazo e nas condições estabelecidas, por serem detentoras de conhecimento e competência em sua área de atuação. Somado a isso, o seu envolvimento nesses tipos de projetos lhes garante à produção e o fornecimento de grandes lotes de componentes e/ou produtos, o que faz parte da natureza do processo de negócio das mesmas.

Para situações onde são necessários baixos volumes de produção, ou produtos de menor complexidade e responsabilidade técnica, a alternativa passa a ser as pequenas e médias empresas (PME). Sob essa visão a inserção de PMEs, na cadeia de fornecedores e/ou parceiros, tem sido uma opção às montadoras para flexibilizar a produção de produtos complexos a custos razoáveis e que necessitem de pequenos volumes. A escolha correta do fornecedor e/ou parceiro é determinante para garantir o sucesso no desenvolvimento de um produto, e para isso são necessários critérios para a definição de quais empresas (pequenas e médias) dispõem de capacidade tecnológica para se tornarem colaboradoras e participarem do PDP.

Entende-se como capacidade tecnológica ou de engenharia a existência de pessoal, ferramentas e sistemas capazes de darem suporte adequado ao desenvolvimento de um produto atendendo requisitos funcionais, de inovação e de qualidade.

Este trabalho tem por objetivo discutir e propor um conjunto de critérios qualificadores, direcionados para empresas de pequeno e médio porte, visando identificar aquelas que estão melhor capacitadas para se tornarem fornecedores e/ou parceiros no desenvolvimento de novos produtos (componentes) automotivos. Os critérios são baseados na avaliação da capacidade tecnológica com relação ao desenvolvimento de produtos, bem como a sua inter-relação com a qualidade do projeto do produto. Os critérios propostos foram testados em uma montadora de ônibus localizada na região serrana do RS, a qual necessita desenvolver produtos 
estratégicos, críticos e complexos e que apresentam elevado valor agregado, mas que necessitam de pequenos volumes de produção.

A próxima seção apresenta uma revisão sobre os critérios para 0 desenvolvimento de fornecedores e seu envolvimento no processo de desenvolvimento de produtos. Na seqüencia é apresentado o método de pesquisa adotado e os critérios elaborados para quantificar a capacidade tecnológica de pequenas e médias empresas se tornarem fornecedores qualificados e participarem do desenvolvimento de novos produtos. Por fim são apresentados os resultados da aplicação dos critérios em 3 empresas, e, finalmente são apresentadas as considerações finais do trabalho.

\section{O DESENVOLVIMENTO DE FORNECEDORES E SEU ENVOLVIMENTO NO PDP}

As empresas montadoras de veículos têm priorizado suas atividades e competências na criação de características-chave que identificarão o produto junto ao mercado consumidor, cabendo aos seus parceiros/fornecedores a responsabilidade sobre o aprimoramento tecnológico dos diferentes sistemas que integram o veículo (KARLSSON, 2003; FREYSSENET e LUNG, 2003; PEREIRA E GEIGER, 2005). Considerando que o parceiro e/ou fornecedor é quem domina a tecnologia vinculada ao componente que produz, não somente no aspecto funcional, mas também do seu processo produtivo, é ele quem melhor pode prover soluções de projeto que facilitem a manufatura e manuseio da peça, uma vez que sejam garantidas as características e propriedades de desempenho no produto final, i.e. veículo.

Segundo Rozenfeld et al. (2006), existem diferentes tipos de envolvimento de um fornecedor no PDP. Essas diferenças são causadas principalmente por fatores de risco tecnológico, financeiro e de inovação (SPEKMAN, KAMAUFF JR e MYHR, 1998). Independente do tipo de envolvimento ele deve acontecer prioritariamente nas fases iniciais do projeto (ESI - Early Supplier Involvement) buscando um resultado mais eficaz do PDP (PIRES, 2004; BIDAULT, DESPRES, BUTLER, 1996; CALVI et al, 2001). 
Para a definição do tipo de envolvimento do parceiro no PDP é necessário selecioná-lo segundo critérios coerentes com a cultura da empresa. Esse tema tem sido objeto de estudo de diversos pesquisadores, dentre os quais se citam Humphrey, Lecler, e Salerno, (2000), Tan e Wisner (2003); Pereira e Geiger (2005) e Assumpção (2003).

Alguns critérios qualificadores no desenvolvimento e seleção de fornecedores usualmente adotados, segundo Lima (2004), Salerno et al. (2003) e Salerno et al (2010), são: a certificação de qualidade, a capacidade financeira, a qualificação do processo produtivo, a capacidade de engenharia e o histórico de fornecimento.

Os diferentes critérios adotados para a seleção de um parceiro, devem se basear em variáveis corporativas, deixando claro ao mercado qual é a sistemática, suas prioridades e exigências (MOTWANI et al, 1999; LIU, DING e LALL 2000; FARIA e VANALLE, 2006, VRIES E HUIJSMAN, 2011).

Considerando a influência de possíveis diferenças culturais e variáveis corporativas Pereira e Geiger (2005), discutem como a complexidade do produto e o volume de produção pode ser determinante na estratégia de desenvolvimento de pequenos e médios fornecedores. O estudo considerou três grupos de grandes empresas automotivas localizados no Rio Grande do Sul, como mostrado na Figura 1. As empresas não dispunham de uma estratégia uniforme para o desenvolvimento de seus fornecedores, apresentando interações (incipientes, medianas e avançadas) e visões (utilitária, conveniência estratégica e de co-responsabilidade) distintas de sua cadeia de fornecedores.

As empresas classificadas no grupo com interação avançada e visão de coresponsabilidade são montadoras de ônibus e tratores. Segundo os autores tal postura advém da grande quantidade de itens apresentado pelo produto final (mais de 1.000), bem como por seus baixos volumes de produção (centenas de produtos finais no período de um ano). Essa condição faz com que tais montadoras precisem interagir intensamente com seus pequenos fornecedores para incrementar, a custos razoáveis, a flexibilidade produtiva em suas linhas de manufatura. 
Figura 1 - Estratégias de desenvolvimento de pequenos fornecedores em função da complexidade do produto final e do volume de produção.

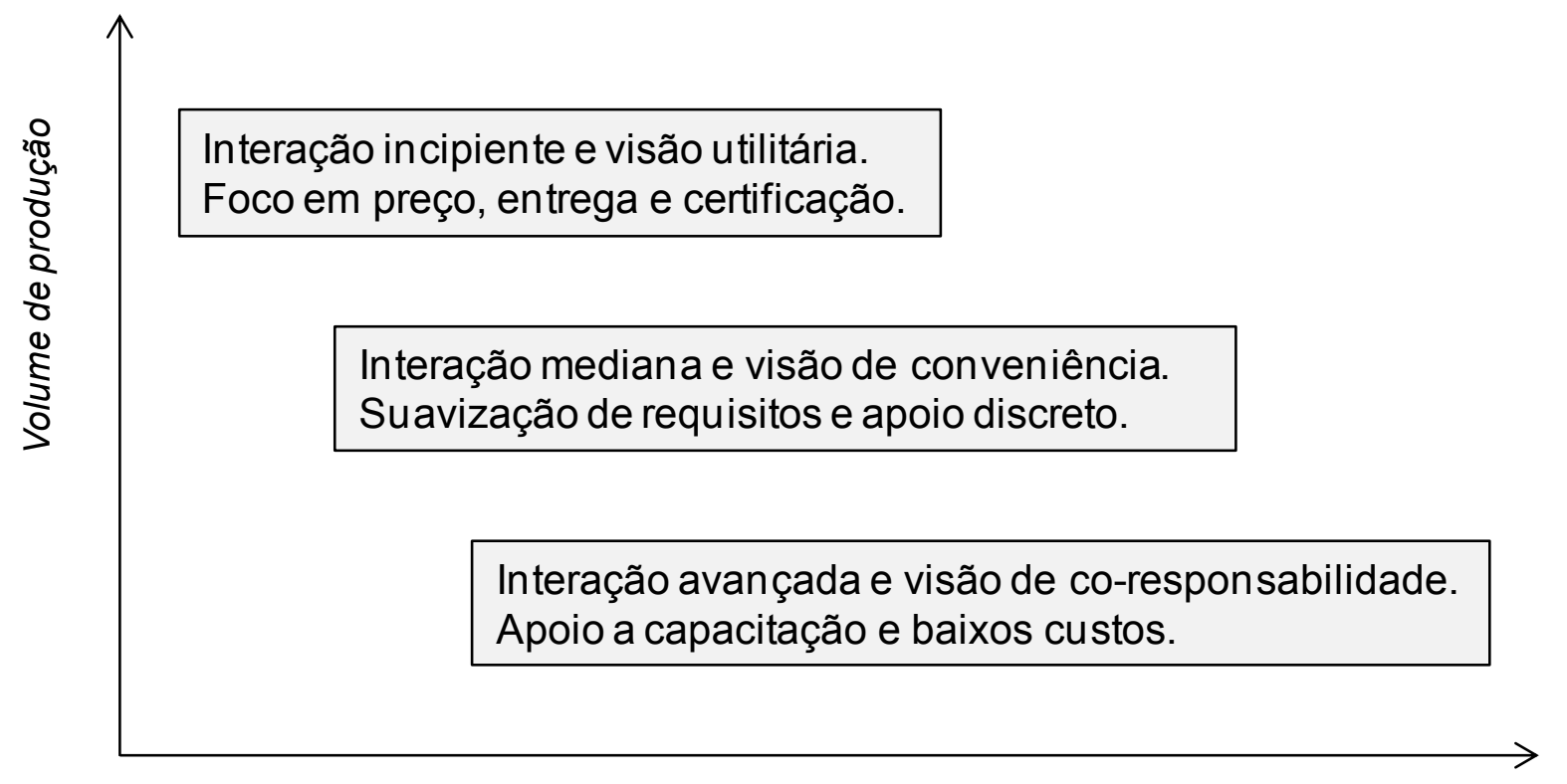

Complexidade do produto final

Fonte: (Adaptado de PEREIRA e GEIGER, 2005)

Além de fatores qualificadores ao fornecimento associados à qualidade do produto e processo, a capacidade financeira e ao histórico de fornecimento (LIMA, 2004; SALERNO et al., 2003; KWON e SUH, 2005, SALERNO et al, 2010), é necessário que essas PME's também passem por um processo de análise de sua competência tecnológica para se tornarem fornecedoras.

\section{O MÉTODO DE PESQUISA}

\subsection{O método empregado e caracterização das empresas envolvidas}

O método de pesquisa empregado pode ser classificado como exploratório, qualitativo e baseado em um estudo de caso durante o qual se realizou, por meio da técnica de observação, um diagnóstico do processo de desenvolvimento de fornecedores em uma empresa montadora de ônibus. O estudo de caso é recomendável para a construção de modelos de análise de mudanças estratégicas organizacionais (MINTZBERG, 1997). Esse método tem por finalidade aprofundar na 
descrição de determinada realidade, caracterizando-se por investigar um fenômeno dentro do seu contexto real, permitindo assim um conhecimento mais apurado do objeto de pesquisa (YIN, 2001).

A montadora de ônibus (cliente) localizada na região serrana do RS é uma empresa líder em seu segmento, produzindo veículos rodoviários e urbanos atendendo tanto ao mercado nacional como internacional. Esta empresa frequentemente necessita desenvolver novos fornecedores para produtos estratégicos e de maior valor agregado e que não são o produto principal, mas que podem ser um diferencial na visão dos clientes, e nesse caso, normalmente os fornecedores são envolvidos no processo de desenvolvimento.

Devido ao fato do volume de produção ser pequeno quando comparado a montadoras de veículos leves ou de passeio, a empresa acaba selecionando PME's como fornecedoras devido a sua flexibilidade e possibilidade de fornecimento de pequenos lotes. Nesse estudo foram avaliadas três empresas de médio porte (fornecedores) que já desenvolvem ou pretendem desenvolver produtos para o segmento automotivo, e que nesse trabalho serão denominados de fornecedor $A, B$ e C.

Esse estudo visa apoiar a escolha da melhor opção para um desenvolvimento conjunto por meio da determinação dos requisitos importantes para um fornecedor no momento de um novo desenvolvimento. Foram avaliados e propostos critérios julgados balizadores na determinação e mensuração da capacidade tecnológica de pequenas e médias empresas se tornarem fornecedoras participantes do processo de desenvolvimento de novos produtos.

\subsection{Definições dos parâmetros de avaliação}

Ao envolver um fornecedor já no início do PDP, a empresa está em busca de capacidades ou soluções que não possui ou domina por completo. Cada produto, ou família de produtos possui particularidades no seu desenvolvimento. Diferentes autores (SALERNO et al, 2010; PEREIRA e GEIGER, 2005; FARIA e VANALLE, 2006; ROZENFELD et al, 2006; ASSUMPÇÃO, 2003) tem destacado como principais motivadores para o envolvimento do fornecedor no PDP os seguintes: o 
conhecimento acerca dos componentes e tecnologia existentes; a melhoria do desempenho funcional; a participação efetiva no refinamento das especificações do projeto; a diminuição do custo do produto; o uso de novos materiais e/ou componentes; a redução do número de alterações e/ou revisões de projeto; a redução na complexidade do projeto, no tempo e custo de desenvolvimento; a melhoria da qualidade e a identificação das tendências tecnológicas do produto.

Considerando que esses são alguns dos principais motivos e resultados que podem ser esperados a partir do envolvimento dos fornecedores no PDP, os mesmos foram considerados na elaboração dos parâmetros para a avaliação da capacidade tecnológica de PMEs fornecedoras.

Nesse estudo, os parâmetros que se destacam foram divididos em três fases associados ao pré-desenvolvimento, desenvolvimento e pós-desenvolvimento do produto. O pré-desenvolvimento está principalmente associado ao conhecimento técnico do pessoal da engenharia que atuará diretamente no projeto do produto. $O$ desenvolvimento está vinculado a estrutura física e aos equipamentos e sistemas disponíveis para a confecção e validação do projeto. O pós-desenvolvimento compreende a capacidade de resolver problemas de projeto que sejam percebidos após o lançamento do produto, bem como suportar o recall de um produto, se for necessário.

Para as três fases foram estabelecidos oito parâmetros relevantes para um fornecedor durante o desenvolvimento de um novo produto, quais sejam: conhecimento técnico; capacitação técnica da engenharia; desenvolvimento de ferramental; capacitação técnica do laboratório de testes; capacitação tecnológica de software; capacidade de inovação; competitividade e assistência técnica, como apresentado na Figura 2.

O primeiro grupo, denominado de parâmetros de pré-desenvolvimento, está associado aos critérios de: conhecimento técnico, capacidade técnica da engenharia e desenvolvimento de ferramental. O segundo grupo, denominado de parâmetros de desenvolvimento, envolve: capacitação técnica do laboratório de testes, capacitação tecnológica de softwares e capacitação a inovação. Por fim, o terceiro grupo (pósdesenvolvimento) avalia os parâmetros de competitividade e assistência técnica. $\mathrm{O}$ item 4 apresenta de modo específico a explicação de cada um dos oito parâmetros e 
suas respectivas alternativas.

Figura 2 - Parâmetros para avaliação tecnológica para PME's.

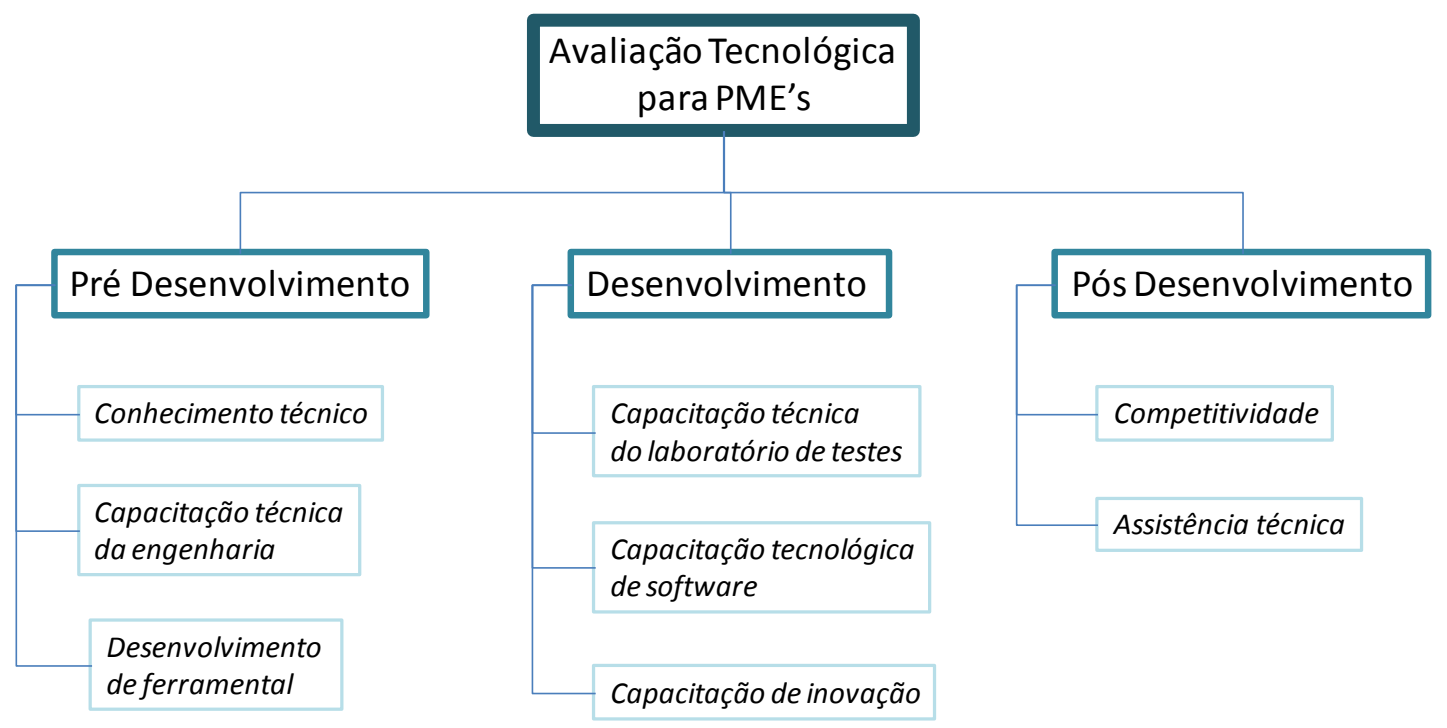

\subsection{Estabelecimento das prioridades dos parâmetros de análise}

A análise hierárquica do processo (AHP) desenvolvida por Saaty (1980) é um método de apoio à decisão (VANDERLEI et al, 2009; DIAS, FERNSTENSEIFER e SELLITTO, 2011). Segundo Morita, Shimizu e Laurindo (1999) o método tem sido empregado para auxiliar as definições de prioridade. Devido a sua aplicação e características julgou-se adequado a sua utilização para definir a importância relativa de cada um dos parâmetros nesse caso estudado. A aplicação, da AHP, pode ser resumida por estruturar uma hierarquia, começando pelo objetivo principal, passando pelos critérios de avaliação e, finalmente chegando ao mais baixo nível da hierarquia, geralmente uma relação de alternativas para cada um dos critérios; e por fim estabelecer as prioridades. A Figura 3 apresenta a organização estrutural adotada nesse trabalho.

Definida a estrutura para a análise hierárquica procura-se estabelecer a importância relativa de cada critério de decisão. São feitas comparações binárias entre os critérios, criando-se uma matriz normalizada que definirá a importância relativa dos parâmetros de análise. 
Figura 3 - Estrutura usada para a definição da importância relativa dos parâmetros de análise

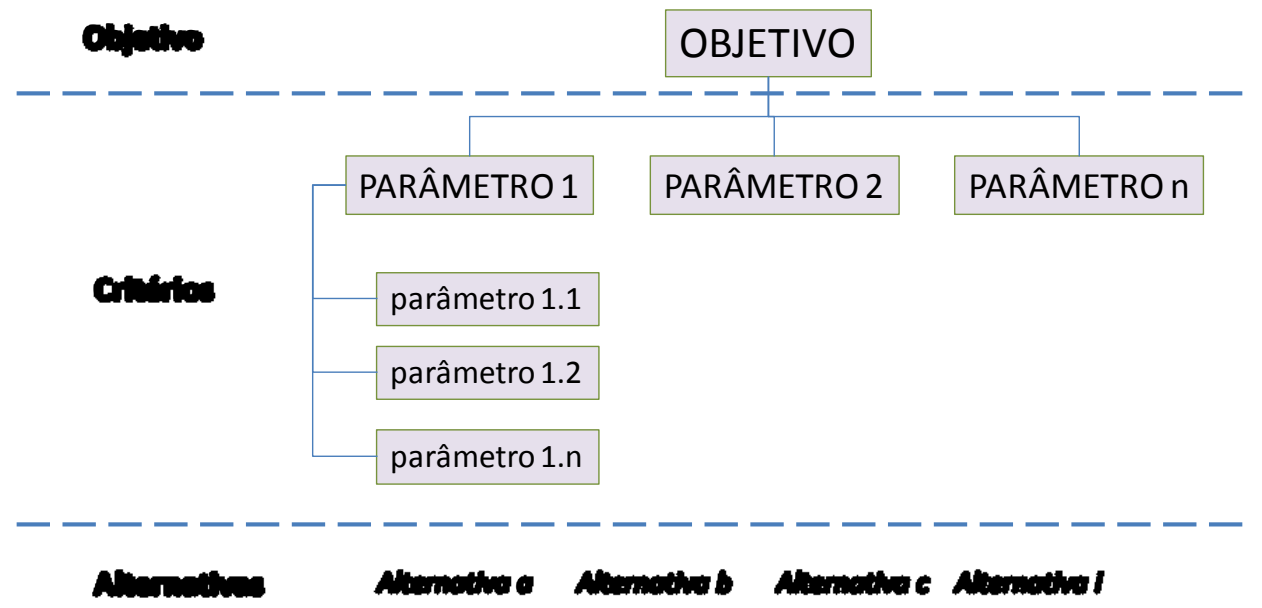

Nesse estudo de caso, a montagem da matriz de comparação foi realizada por integrantes da engenharia de desenvolvimento e de pessoas ligadas ao setor de desenvolvimento de fornecedores, considerando as diretrizes determinadas pela alta administração da empresa. Com o isso foi possível estabelecer a preferência de cada critério par a par, conforme mostrado na Figura 4.

Figura 4 - Matriz de comparação e hierarquização dos parâmetros de análise

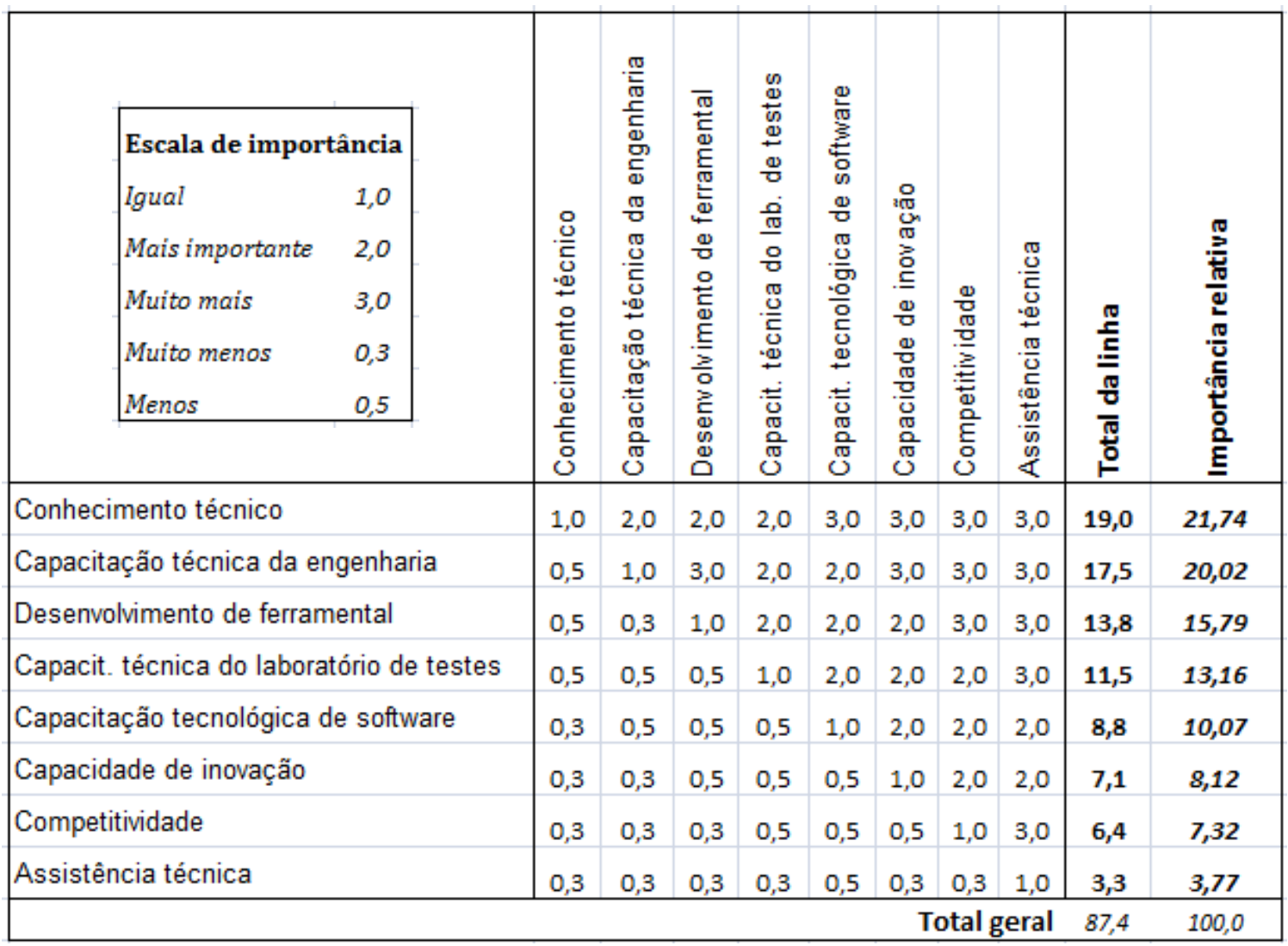

Revista Produção Online, Florianópolis, SC, v.13, n. 2, p. 577-600, abr./jun. 2013. 


\subsection{Determinação do valor mínimo para a seleção dos fornecedores}

Estabelecidos os parâmetros para a avaliação é necessário determinar o peso de cada item para produzir a nota final do fornecedor referente à sua capacitação. Os parâmetros foram divididos em 5 grupos considerando o valor da importância relativa (Ir) determinado na matriz de comparação (Figura 4). A Tabela 1 apresenta os pesos para cada um dos oito parâmetros.

Tabela 1 - Peso relativo dos parâmetros de avaliação

\begin{tabular}{|c|c|c|c|}
\hline & $\mathrm{Pe}$ & Importância & Parâmetros \\
\hline so & \multicolumn{2}{|c|}{ relativa } & \\
\hline & \multirow{2}{*}{5} & \multirow{2}{*}{ Ir $>20$} & Conhecimento Técnico \\
\hline & & & Capacitação Técnica da Engenharia \\
\hline & \multirow[t]{2}{*}{4} & \multirow[t]{2}{*}{$15<\operatorname{lr}<20$} & Desenvolvimento de Ferramental \\
\hline & & & Capacitação Técnica do Laboratório \\
\hline & \multirow[t]{2}{*}{3} & \multirow[t]{2}{*}{$10<\operatorname{lr}<15$} & de Testes \\
\hline & & & Capacitação Tecnológica de Software \\
\hline & \multirow{2}{*}{2} & \multirow{2}{*}{$5<I r<10$} & Capacidade de Inovação \\
\hline & & & Competitividade \\
\hline & 1 & $5<I r$ & Assistência Técnica \\
\hline
\end{tabular}

Cada um dos oito parâmetros avaliados pode ser caracterizado em uma escala de zero a quatro, ou seja, 5 níveis de escolha, semelhante ao proposto na escala Likert (PEDROZO et al, 2010), para a verificação de cada quesito, conforme mostrado na Tabela 2. A escala zero representa a pior condição, ou seja, a empresa não possui condições mínimas ou suficientes de realizar o desenvolvimento do produto. O valor um na escala mostra que a empresa ainda está abaixo do nível esperado, devendo qualificar as pessoas, os métodos e a estrutura existente. $\mathrm{O}$ valor dois significa que a empresa atende aos requisitos mínimos de capacidade tecnológica, mas deve buscar melhorias em seu PDP considerando um processo de melhoria contínua. $O$ valor 3 representa boas condições de desenvolvimento normalmente acima daquilo que é suficiente, enquanto a escala quatro representa a condição ideal da empresa, considerando o tipo de produto e setor em estudo. 
Tabela 2 - Significado das notas de cada uma das alternativas para os parâmetros de avaliação.

\begin{tabular}{ll}
\hline Notas & Significado da nota do quesito \\
\hline 4 & Condição ideal \\
3 & Situação intermediária, acima do suficiente \\
2 & Atende aos requisitos de modo suficiente \\
1 & Situação intermediária, abaixo do suficiente \\
0 & Não possui condições suficientes \\
\hline
\end{tabular}

Para determinar a nota final do fornecedor é necessário multiplicar o peso de cada requisito (determinado pela importância relativa, Tabela 1) pelo escore obtido na avaliação de cada um dos parâmetros (Tabela 2). A Equação 1 mostra as variáveis para a determinação da nota final.

$N_{F}=\left(5 \times N_{1}\right)+\left(5 \times N_{2}\right)+\left(4 \times N_{3}\right)+\left(3 \times N_{4}\right)+\left(3 \times N_{5}\right)+\left(2 \times N_{6}\right)+\left(2 \times N_{7}\right)+\left(1 \times N_{8}\right)$

Onde:

$N_{F}-$ nota final

$N_{1}$ - escore do Conhecimento Técnico.

$\mathrm{N}_{2}$ - escore da Capacitação Técnica (Engenharia).

$\mathrm{N}_{3}$ - escore do Desenvolvimento de Ferramental.

$\mathrm{N}_{4}$ - escore da Capacitação Técnica (Laboratório).

$\mathrm{N}_{5}$ - escore da Capacitação Técnica (Software).

$N_{6}$ - escore da Capacidade de Inovação.

$N_{7}$ - escore da Competitividade.

$N_{8}$ - escore da Assistência Técnica.

Devido às características do produto final as empresas precisam desenvolver itens de baixa complexidade em um curto prazo, bem como, produtos complexos que exigem ferramentais especiais e que necessitam, para tanto, de um maior tempo. Sabe-se que a exigência técnica do fornecedor para desenvolver um produto está diretamente relacionada a complexidade do projeto, portanto produtos com um nível de exigência técnica maior requerem fornecedores mais preparados. 
Neste trabalho os componentes da empresa foram divididos em três diferentes graus de complexidade: alta, média e baixa. Para tanto, foi analisada por uma equipe da engenharia de desenvolvimento a importância técnica do componente no produto final (ônibus), o grau de exigência tecnológica do produto, a necessidade de desenvolvimento de ferramental, entre outras características.

Como exemplo de um item de alta complexidade pode-se citar os conjuntos de lanternas (traseiras, laterais ou dianteiras), que necessitam de uma série de ferramentais de produção, bem como deve atender à normas internacionais de segurança. Outros exemplos de itens de alta complexidade são: sistema de ar condicionado, geladeiras e peças injetadas de alumínio, entre outros.

Peças extrudadas em ABS, Acrílico e PVC são consideradas de média complexidade, pois requerem ferramentais de simples confecção e as peças são de fácil produção exigindo apenas controle do processo e do material. Peças fabricadas pelo processo de vacuum forming são consideradas de fácil desenvolvimento devido à simplicidade e rapidez na confecção de ferramentas, bem como a facilidade de produção das peças finais. Cabe salientar que as definições aqui adotadas se referem a realidade tecnológica do setor e da empresa, podendo se diferenciar para outras realidades.

Como critério estabelecido nesse trabalho, durante o processo de avaliação dos fornecedores, esses não poderão obter nota igual a zero em qualquer parâmetro sob a penalidade de serem automaticamente considerado inaptos a participar do desenvolvimento do produto ou peça, mesmo que a composição final de sua nota fique acima do mínimo exigido.

Para os itens de complexidade alta, onde o nível de exigência técnica é elevado, dentre os dois critérios de avaliação com peso 5 , que medem 0 conhecimento técnico e a capacitação técnica da engenharia, pelo menos um deve ter a nota máxima, que é equivalente à nota 4 , e o outro deve ser pelo menos nota 3 . Para os demais critérios é desejável que o fornecedor atinja a nota 3. A nota mínima de 80 pontos deve ser obtida pelo fornecedor de produtos com grau de complexidade elevada (Tabela 3). Caso não seja necessário o desenvolvimento de ferramental, desconsidera-se este requisito e a nota mínima do fornecedor será de 68 pontos.

Revista Produção Online, Florianópolis, SC, v.13, n. 2, p. 577-600, abr./jun. 2013. 
Para os itens de média complexidade, é necessário que o fornecedor possua no mínimo nota 3 para os requisitos técnicos, que são conhecimento técnico, capacitação técnica da engenharia, desenvolvimento de ferramental, capacitação técnica do laboratório e capacitação tecnológica de software. Para os demais requisitos é desejável que o fornecedor atinja a nota 2. A nota mínima para os fornecedores de média complexidade é 70 .

Tabela 3 - Pontuação mínima em função do grau de complexidade do produto

\begin{tabular}{|c|c|c|c|c|c|}
\hline Grau & de & Nota & Nota & mínima & sem \\
\hline complexidade & & mínima & ferramental & & \\
\hline Alta & & 80 & 68 & & \\
\hline Média & & 70 & 58 & & \\
\hline Baixa & & 64 & 52 & & \\
\hline
\end{tabular}

Para os fornecedores onde o grau de complexidade de desenvolvimento é baixo, deseja-se pelo menos nota 3 para os critérios de conhecimento técnico, capacitação técnica da engenharia e desenvolvimento de ferramental. Para os demais critérios, é necessário pelo menos a nota 2. A nota mínima de 64 para os itens de baixa complexidade de desenvolvimento. Caso não seja necessário o desenvolvimento de ferramental, deve-se desconsiderar este requisito e a nota mínima será de 52 pontos.

\section{PARÂMETROS PARA A AVALIAÇÃO DA CAPACIDADE TECNOLÓGICA DO FORNECEDOR}

Conhecimento Técnico: este parâmetro tem o intuito de verificar se 0 fornecedor possui o conhecimento efetivo de sua linha de produtos sendo capaz de projetar um sistema somente a partir de informações primárias (necessidade de aplicação, por exemplo). O conhecimento pleno de sua atividade essencial faz com que o fornecedor consiga observar possíveis falhas de projeto durante as fases iniciais, evitando gastos e retrabalhos em ferramentais e protótipos. A escala de avaliação para este requisito é:

0 - Não possui conhecimento técnico do produto, somente detêm Revista Produção Online, Florianópolis, SC, v.13, n. 2, p. 577-600, abr./jun. 2013. 
conhecimento sobre processo de produção.

1 - Produz o produto especificado sem capacidade de realizar questionamentos referentes ao projeto do produto.

2 - Possui capacidade de questionar possíveis erros de especificações de projeto.

3 - Possui capacidade de questionar possíveis erros de especificações de projeto e propor soluções.

4 - Possui capacidade plena de elaborar especificações e produzir um produto a partir de uma necessidade de aplicação do cliente.

Capacitação Técnica da Engenharia: o objetivo desse parâmetro é detectar se o fornecedor possui recursos humanos com competência técnica (cursos de qualificação em diferentes níveis) para projetar um produto e propor alternativas de desenvolvimento. Para verificar a capacidade técnica do departamento de engenharia será realizada uma análise do quadro funcional técnico da empresa fornecedora. A avaliação proposta ficou da seguinte forma:

0 - Não possui departamento de engenharia.

1 - Não possui departamento de engenharia, somente profissionais que interpretem desenhos.

2 - Não possui departamento de engenharia, porém existem profissionais com formação técnica.

3 - Possui departamento de engenharia com profissionais capacitados para propor alternativas de desenvolvimento.

4 - Possui departamento de engenharia com profissionais capacitados para o desenvolvimento pleno de um produto.

Desenvolvimento de Ferramental: o conhecimento do fornecedor no projeto e confecção de ferramentais é um item importante durante o desenvolvimento de um novo produto. Uma concepção incorreta das ferramentas pode ocasionar problemas de qualidade na peça, bem como pode não atender a demanda de produção. Os prazos de desenvolvimento são em sua grande maioria determinados a partir do tempo necessário para projeto e execução dos ferramentais. Para verificação deste 
quesito determinou-se a seguinte escala:

0 - Não realiza projeto e nem produz ferramental.

1 - Não realiza projeto e nem produz ferramental, porém executa em empresas terceirizadas.

2 - Não realiza projeto e nem produz ferramental, porém executa em empresas terceirizadas intervindo com informações e conhecimentos técnicos.

3 - Realiza o projeto do ferramental, porém o mesmo é confeccionado por uma empresa terceirizada.

4 - Realiza o projeto e a confecção do ferramental em sua plenitude.

Capacitação Técnica do Laboratório de Testes: a realização de testes é um quesito importante a ser avaliado. O fornecedor deve ter conhecimento de quais serão as solicitações de uso a que seu produto será submetido, para tanto se faz necessário a realização de ensaios e testes preliminares. Para mensurar esta capacidade foram determinada a seguinte escala:

0 - Não possui laboratório de testes.

1 - Não possui laboratório de testes, porém executa testes em empresas externas.

2 - Não possui laboratório de testes, porém existem mecanismos internos de testes de produtos, sendo a homologação final realizada por terceiros.

3 - Possui laboratório de testes capaz de homologar uma grande parcela de sua linha de produtos.

4 - Possui laboratório de testes capaz de homologar toda a sua linha de produtos.

Capacitação Tecnológica de Software: pretende-se verificar com este parâmetro quais são os recursos computacionais que o fornecedor possui e que podem auxiliá-lo no processo de desenvolvimento de um novo produto. Basicamente são analisados quais os programas de CAE/CAD são utilizados por sua engenharia. O parâmetro será avaliado da seguinte maneira:

0 - Não possui software de CAE/CAD. 
1 - Não possui software de CAE/CAD, porém uma empresa terceirizada executa e lê seus desenhos.

2 - Possui somente software de leitura de desenhos, sendo que uma empresa terceirizada realiza seus desenhos.

3 - Possui software de CAD em 2D (leitura e execução).

4 - Possui software de CAE/CAD 3D.

Capacidade de Inovação: pretende-se verificar se o fornecedor possui alguma tecnologia (produto e/ou processo) que seja inovadora. A capacidade do fornecedor em desenvolver produtos inovadores agregará um valor final significativo ao produto, sendo que um dos meios de avaliação desse critério, por exemplo, pode ser a existência ou não de patentes industriais. A escala de valores foi estabelecida da seguinte maneira:

0 - Não possui produto ou tecnologia inovadora.

1 - Não possui produto ou tecnologia inovadora, porém possui convênio com instituições que estimulam programas de inovação.

2 - Não possui produto ou tecnologia inovadora, porém participa, ou já participou de programas de inovação.

3 - Participou em associação com outra empresa na concepção de um produto ou tecnologia inovadora.

4 - Realizou e ou executou o projeto de um produto inovador.

Competitividade: tem como objetivo verificar se a empresa fornecedora já faz parte da cadeia de suprimentos do mercado de ônibus, e qual sua posição competitiva em relação aos seus concorrentes. A avaliação deste quesito é importante, pois o mercado de ônibus possui algumas particularidades, como por exemplo, o elevado grau de customização e a necessidade de rapidez nos desenvolvimentos. $O$ fato de o fornecedor já atuar com clientes com estas características, certamente auxiliará no momento do desenvolvimento de um novo produto. Também é considerada uma boa referência o fornecimento de peças para empresas fabricantes de automóveis, devido ao padrão de exigência técnica e de qualidade solicitada por este mercado. Para a avaliação deste quesito usa-se a 
seguinte graduação:

0 - Nunca forneceu para o mercado de ônibus ou automotivo.

1 - Participa indiretamente no fornecimento de produtos para o mercado de ônibus ou automotivo.

2 - Participa no fornecimento direto de produtos para uma pequena parcela do mercado de ônibus ou automotivo.

3 - Participa no fornecimento direto de produtos para uma grande parcela do mercado de ônibus ou automotivo.

4 - Participa no fornecimento direto de produtos para uma grande parcela do mercado de ônibus ou automotivo, sendo na sua maioria o fornecedor principal.

Assistência Técnica: pretende-se verificar o comportamento de seu produto no mercado e qual a capacidade em resolver possíveis problemas. Pesquisar o índice de problemas do fornecedor com Assistência Técnica é um bom indicador para avaliar os riscos de desenvolvimento. Para medir este quesito adotaram-se os seguintes valores:

0 - Não possui departamento de Assistência Técnica.

1 - Não possui departamento de Assistência Técnica, porém existem profissionais de outras áreas que dão suporte técnico.

2 - Possui departamento de Assistência Técnica com histórico frequente de reclamação de mercado.

3 - Possui departamento de Assistência Técnica com baixa frequência de reclamação de mercado.

4 - Possui departamento de Assistência Técnica sem histórico de reclamação de mercado.

\section{VALIDAÇÃO DO MODELO PROPOSTO EM TRÊS FORNECEDORES}

Para realizar a validação do método proposto, foram escolhidos três fornecedores da mesma linha de produtos, cujo grau de complexidade de 
desenvolvimento de seus itens foi considerado alto.

A avaliação da capacitação tecnológica destes fornecedores foi realizada em duas etapas. Na primeira, o questionário foi enviado para os fornecedores e os mesmos responderam aos oito quesitos propostos. Obteve-se, portanto uma autoavaliação dos fornecedores referente à sua capacitação tecnológica. Na segunda etapa, os fornecedores foram visitados, e a avaliação foi realizada por um engenheiro integrante do setor de desenvolvimento de fornecedores da montadora. Uma vez os questionários respondidos foi possível conhecer a nota de cada um dos fornecedores.

A nota final do fornecedor A pela análise da montadora é menor do que a nota obtida pela auto-avaliação. A diferença de valor foi em função da avaliação da capacitação técnica do laboratório. A nota mínima exigida para um fornecedor de um item de alto grau de complexidade é 80 pontos, e, portanto este fornecedor está apto à participar de um projeto de desenvolvimento de produto desde as fases inicias de acordo com a avaliação proposta, como pode ser visto na Figura 5.

Para o fornecedor B é possível observar que a nota final pela análise da montadora é maior do que a nota obtida na auto-avaliação. A diferença deve-se somente à diferença de avaliação da capacidade técnica do laboratório. Como o fornecedor B obteve a nota final de 83 pontos, encontra-se apto a participar das fases iniciais de um PDP com a montadora. A nota final do fornecedor $C$ pela autoavaliação foi de 71 pontos. Este valor é insuficiente para que um fornecedor seja considerado apto a participar desde as fases iniciais de um projeto de desenvolvimento de produto, porém a nota final obtida pela avaliação da montadora foi de 81 pontos, como pode ser visto na Figura 5.

Esta diferença se deve à avaliação da capacitação técnica da engenharia e do conhecimento técnico, sendo que estes dois quesitos são os mais relevantes para a formulação da nota final. Associado a isso, o fornecedor C possui como clientes várias empresas do ramo automobilístico, cuja exigência técnica é grande e talvez por este motivo a sua análise tenha sido um pouco mais rígida do que a análise feita pela montadora.

A análise das notas obtidas pela avaliação da montadora, determina que os três fornecedores estão aptos à participar de um projeto de desenvolvimento de 
produto de alta complexidade desde as fases iniciais. Entretanto, percebeu-se diferentes interpretações (empresa e fornecedores) sobre alguns parâmetros da avaliação.

Figura 5 - Comparativo entre a nota final da auto-avaliação e a nota final da avaliação.

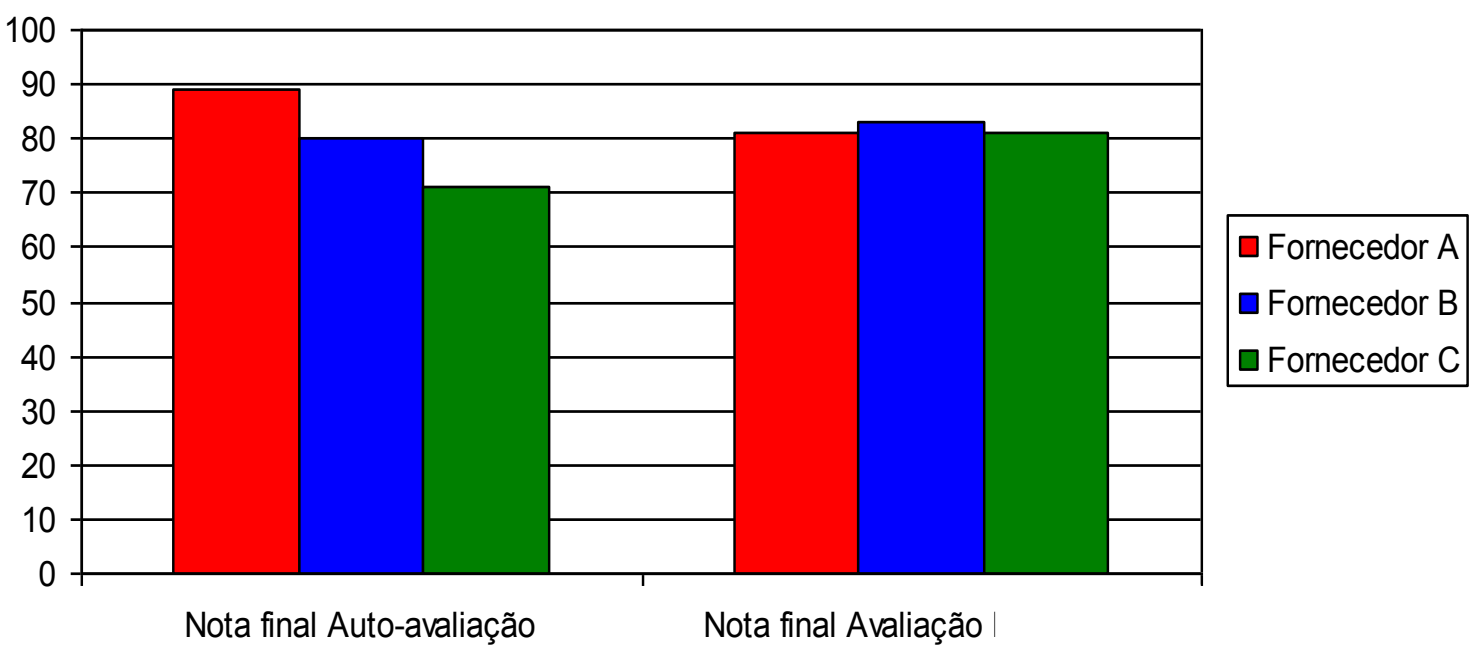

Tal fato é possível, pois mesmo se estabelecendo critérios estes podem ser interpretados de formas diferentes, por estarem descritos de forma qualitativa e não quantitativa. Além disso, é necessário se estabelecer um padrão de linguagem permitindo um entendimento mais uniforme sobre cada um dos quesitos avaliados.

\section{CONSIDERAÇÕES FINAIS}

Este trabalho procurou estabelecer parâmetros que permitam avaliar a capacidade tecnológica de uma PMEs ser colaboradora no desenvolvimento de um produto e posteriormente fornecê-lo. Foram utilizados alguns conceitos de PDP e SCM na elaboração do procedimento.

A determinação dos requisitos importantes a serem avaliados no fornecedor, está diretamente associada às características peculiares do produto final. Neste trabalho foram criados oito parâmetros que envolvem características do prédesenvolvimento, desenvolvimento e pós-desenvolvimento.

Foram considerados diferentes pesos para os parâmetros que permitem obter 
uma nota final que indica se o fornecedor pode desenvolver produtos de baixa, média ou alta complexidade.

$\mathrm{Na}$ análise das notas dos três fornecedores avaliados, percebeu-se que os valores ficaram muito próximos devido ao fato de os mesmos terem características semelhantes. É importante que seja realizada uma análise em fornecedores com capacidades tecnológicas distintas para verificar se é possível medir esta diferença.

Observaram-se também diferentes interpretações para os parâmetros elaborados. Esse fato merece uma maior atenção na tentativa de se buscar meios que tornem a avaliação menos qualitativa e mais quantitativa.

A existência de um procedimento quantitativo deve originar a criação de um "modelo de maturidade" que consiga classificar o fornecedor quanto a sua capacidade de atender adequadamente diferentes níveis de complexidade de desenvolvimento de produtos.

\section{REFERÊNCIAS}

AMARAL, D.C., TOLEDO, J.C. Colaboração cliente-fornecedor no processo de desenvolvimento de produto. Gestão \& Produção v.7, n.1, p. 56-72, abr. 2000.

ASSUMPÇÃO, M. R. P. Reflexão para gestão tecnológica em cadeias de suprimento. Gestão \& Produção, v.10, n.3, p.345-361, dez. 2003.

BALLOU, R. H. Business logistics/supply chain management: planning, organizing, and controlling the supply chain. 5.ed. Upper Saddle River, N.J.: Pearson Prentice Hall, 2004.

BIDAULT, F.; DESPRES, C.; BUTLER, C. New product development and early supplier involvement (ESI): the drivers of ESI adoption, In: Proceeding of the Product Development Management Association International Conference. Orlando, 1996.

CALVI, R.; LE DAIN, M.; HARBI, S.; BONOTTO, V. I. How to manage Early Supplier Involvement (ESI) into the New Product Development Process (NPDP): several lessons from a French study. In: Proceedings of the 10th International Annual IPSERA Conference. Jönköping, Suécia, 2001.

CHIESA, V.; MANZINI, R.; TECILLA, F. Selecting sourcing strategies for technical innovation: an empirical case study. International Journal of Operations \&

Production Management, Bradford, v. 20, n. 9, p. 1017-37, set. 2000. 
COUSINS, P.; SPEKMAN, R. Strategic supply and the management of inter-and intra-organisational relationships. Journal of Purchasing and Supply Management. Rotterdam, v. 9, n. 1, p. 19-29, jan. 2003.

DIAS, M. F. P.; FERNSTENSEIFER, J. E.; SELLITTO, M. A. Análise multicriterial em estratégia de operações: estudo de caso com compradores de arroz de seis redes supermercadistas. Revista Produção Online, Florianópolis, v. 11, n. 3, p. 707-734, set. 2011. ISSN 16761901. Disponível em:

<http://www.producaoonline.org.br/rpo/article/view/669>. Acesso em: 12 jul. 2012.

FARIA, P. O.; VANALLE, R. M. Critérios para a seleção de fornecedores: uma análise das práticas de grandes empresas industriais do Estado do Espírito Santo. In: ENEGEP, 26,. 2006. Anais... Fortaleza, CE, Brasil, 2006.

FREYSSENET, M.; LUNG, Y. Perspectives of the car industry: How to draw them after the numerous denials imposed on the previous forecasts?, La Lettre du Gerpisa, ÉVRY Cedex, v.9, n. 171, p. 7-12, out. 2003.

HONG, Y. C. Gestão de estoques na cadeia de logística integrada: supply chain. 3.ed. São Paulo: Atlas, 2006. 220 p.

HUMPHREY, J.; LECLER, Y.; SALERNO, M. S. Global strategies and local realities: the auto industry in emerging markets. 1. ed. London: Macmillan, 2000. $300 \mathrm{p}$.

KARLSSON, C. The development of industrial networks. Journal of Operations and Production Management, Bradford, v. 23, n. 1, p. 44-61, jan. 2003.

KWON, IK-W. G.; SUH, T.; Trust, commitment and relationships in supply chain management: a path analysis. Supply Chain Management: An International Journal, v.10, n. 1, p. 26-33, 2005 .

LIMA, J. C. S. Um Estudo sobre a reconfiguração da função compras em empresas do setor automotivo. Tese (Doutorado em Engenharia de Produção) Escola Politécnica, Universidade de São Paulo, São Paulo, 2004. Disponível em: <http://www.usp.br>.

LIU J.; DING F. Y.; LALL V. Using Data Envelopment Analysis to Compare Suppliers for Supplier Selection and Performance Improvement. Supply Chain Management: An International Journal, v. 5, n. 3, p. 143-150, March 2000.

MINTZBERG, H. An emerging strategy of "direct" research. Administrative Science, p. 582-589, 1997,

MORITA, H.; SHIMIZU, T.; LAURINDO, F. J. L. Modelos para estruturar e avaliar alternativas de decisão em Tecnologia da Informação. In: ENCONTRO NACIONAL 
DE ENGENHARIA DE PRODUÇÃO E V CONGRESSO INTERNACIONAL DE ENGENHARIA INDUSTRIAL. 19,. 1999. Anais... Rio de Janeiro, 1999. 1 CD-ROM.

MOTWANI J.; YOUSSEF M.; KATHAWALA Y.; FUTCH E. Supplier selection in developing countries: a model development. Integrated Manufacturing Systems, v. 10, n. 3, p. 154-162, March 1999.

PEDROSO, B.; PILATTI, L. A.; SANTOS, C. B.; SANTOS Jr, G. Potencial motivador do trabalho: tradução e adaptação cultural do instrumento de Hackman e

Oldham. Revista Produção Online, Florianópolis, v. 10, n. 3, p. 670-697, ago. 2010. Disponível em: <http://www.producaoonline.org.br/rpo/article/view/533>. Acesso em: 12 Jul. 2012.

PEREIRA, G. M.; GEIGER, A. Complexidade do produto e volume de produção como determinantes da estratégia de desenvolvimento de fornecedores automotivos. Gestão \& Produção, v.12, n.2, p.191-201, maio/ago. 2005

PIRES, S. R. I. Gestão da cadeia de suprimentos. São Paulo: Atlas, 2004.

ROZENFELD, Henrique; FORCELLINI, Fernando Antônio; AMARAL, Daniel Capaldo; TOLEDO, José Carlos de; SILVA, Sergio Luis da; ALLIPRANDINI, Dário Henrique; SCALICE, Régis Kovacs. Gestão de desenvolvimento de produtos: uma referência para a melhoria do processo. 1. ed. São Paulo: Editora Saraiva, 2006.

SAATY, T.L The analytic Hierarchy Process. McGraw-Hill: New York, 1980.

SALERNO, M. S., MARX, R., ZILBOVICIUS, M. A nova configuração da cadeia de fornecimento na indústria automobilística do Brasil. Revista de Administração da USP, v. 38, n. 3, p. 192-204, 2003.

SALERNO, M. S., MIRANDAB, Z., KAMISAKIC, F. Y., MALUTAD, G. Alavancando pesquisa, desenvolvimento e inovação no setor de autopeças: análise e propostas a partir de survey e estudo qualitativo focado. Revista Produção v. 20, n. 4, p. 565575,2010

SPEKMAN, R. E.; KAMAUFF JR, J. W.; MYHR, N.; An empirical investigation into supply chain management: a perspective on partnerships. Supply Chain Management, v.3, n. 2, p. 53-67,1998.

TAN, K.; WISNER, J. A study of operations management constructs and their relationships. International Journal of Operations \& Production Management, Bradford, v. 23, n. 11, p. 1300-25, nov. 2003.

VANDERLEI, M. L.; MUNIZ Jr, J.; MARINS, F .A. S., MIRANDA, G. W. A. Implantação de controle baseado no sistema de execução da manufatura (mes): 
análise em empresa de usinagem no setor aeronáutico. Revista Produção Online, Florianópolis, v. 9, n. 4, dez. 2009. Disponível em:

<http://www.producaoonline.org.br/rpo/article/view/380>. Acesso em: 16 jun. 2012.

VRIES, J.; HUIJSMAN, R.; Supply chain management in health services: an overview. Supply Chain Management: An International Journal, v. 16, n. 3, p. 159-165, 2011.

YIN, R. K. Estudo de caso: planejamento e métodos. 2.ed. Porto Alegre: Bookman, 2001.

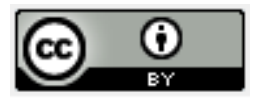

Artigo recebido em 05/12/2011 e aceito para publicação em 0304/2013.

Revista Produção Online, Florianópolis, SC, v.13, n. 2, p. 577-600, abr./jun. 2013. 